\title{
Robotic screening of intestinal drug absorption
}

A robotic culture system for the high-throughput analysis of drug transport in porcine gastrointestinal tissue explants accurately predicts the absorption of orally taken drugs in the human gut.

\author{
Abdul W. Basit, Christine M. Madla and Francesca K. H. Gavins
}

Predicting how drugs are absorbed in the gastrointestinal (Gl) tract after their oral administration is complex ${ }^{1}$. First, variability among individuals in their gut motility, intestinal enzymes, bile salts, bacteria, $\mathrm{pH}$ range and ingested food can affect the drug's behaviour (such as drug dissolution) in the gut's lumen. Second, the drug must then cross the gut wall whilst battling hostile barriers such as mucus, efflux transporters (membrane proteins that move potentially toxic molecules out of cells and back into the gut) and metabolizing enzymes (Fig. 1), which can also alter the bioavailability of the drug (that is, the fraction of the administered dose that reaches the systemic circulation). Mimicking the GI absorption of drugs with effective in vitro models is thus notoriously difficult. Monolayers of Caco-2 cells on permeable transwell supports are commonly used to model intestinal permeability, owing to their similarities to enterocytes (the absorptive cells that line the intestines), and because they have been extensively characterized and are easy to culture ${ }^{2}$. However, the usefulness of the Caco-2 transwell system for predicting in vivo gut absorption is limited by the absence of other cell types in the epithelium, as well as by the lack of mucus, bile acids and phospholipids that normally contribute to in vivo drug performance ${ }^{3}$. Reporting in Nature Biomedical Engineering, Giovanni Traverso, Robert Langer and colleagues now show that a robotically handled culture system using intact porcine intestinal tissue enables the high-throughput assessment of the intestinal transport of oral drugs ${ }^{4}$.

Traverso and co-authors' culture system involves explanted porcine jejunal tissues sealed over a middle well-plate positioned between an upper and lower plate (Fig. 2a,b). The system can use commercially available 96-well plates or customized well-plates (with $24,48,96,384$ or 1536 wells) that are fully compatible with a robotic instrument, which includes a liquid-handling station, automated well-plate storage, a well-plate reader, and a 6-axis robotic arm enabling plate analysis and transfer without disassembly (Fig. 2c). To calculate the rate of drug transport through the explanted intestinal tissue, the drug is added to the upper plate, and the concentration difference between the upper and lower plates is measured over time. The authors optimized the cultivation of porcine Gl tissue, which was chosen because of its similarities to the human GI anatomy and because of extensive research into the pig's genome ${ }^{5,6}$. Younger porcine GI tissue with an intact stroma layer, cultured in advanced Dulbecco's Modified Eagle media F-12, ensured tissue viability and function for a few weeks. Functionality was confirmed by the gene-expression and protein-expression levels of a wide range of cell-type markers (in particular, Wnt3a, which is essential for the maintenance of intestinal stem cells).

Most cell cultures require routine maintenance every three days to ensure cell viability and function. Traverso and coauthors, however, observed that after one week of culture the tissue explants did not display significant changes in tissue integrity. The longer culture time probably results from the ex vivo maintenance of intestinal stem cells by the stromal layer, as the removal of this section prior to tissue explanting resulted in the loss of the viability of the epithelial layer within 5 days. However, beyond one week of culture there were histological and cell-marker changes with respect to freshly isolated tissue. This suggests that there were adverse effects on cell morphology and function. Also, the analysis of mucin-2 expression suggested that the explant culture reduced the number of mucus-producing goblet cells. Mucus can act as an integral defence system to drugs permeating the GI wall, and therefore its role in impeding oral-drug absorption may not be adequately reflected in the cultured explants ${ }^{6}$. As the authors suggest, further research into the role of mechanical stimulation and the integration of a blood supply via a transport system could extend the lifespan of the cultured explant.

In order to validate intestinal drug absorption, Traverso and co-authors used the culture system to analyse the intestinal transport of 55 drugs with available human pharmacokinetic data. The system predicted the absorption of the 55 drugs with a Spearman correlation of 0.906 . In comparison, the Spearman coefficient for the Caco-2 transwell system was 0.302 (on the basis of published drug-transport values). Computational modelling by using absorption, distribution, metabolism, excretion, and toxicity activity-structure relationsips led to poor correlations. This was is expected, as in the Caco-2 model the tight junctions for paracellular uptake and the proteins for active drug uptake or drug efflux (in particular, ATP-binding cassette transporters and solute carriers) are differently expressed in native human intestinal tissue. Moreover, Caco-2 cells are incompatible with intestinal fluids containing digestive by-products, mucus and bacteria $^{7}$. Instead, the porcine explants preserve and mimic intestinal morphology and the function of metabolizing enzymes and drug transporters, which enables drug-transport experiments in the presence of native intestinal fluid. In addition, drug-food interactions could be explored in this system. Furthermore, the average coefficient of variation of the drug-permeability values for Caco-2 transwells was two times higher for actively absorbed drugs than for passively absorbed drugs, suggesting that the Caco-2 model better predicts in vivo performance for passively transported drugs $^{8,9}$. Yet previous studies have suggested that, owing to genomic instability, Caco-2 cells show variable expression levels of drug transporters, which limits the system's reliability for drug-transport studies.

To showcase the fully automated screening capabilities of the robotic system and its potential for oral-drug development, Traverso and colleagues used it to screen for 2,930 formulations of the model peptide drug oxytocin (chosen for its poor oral bioavailability) with combinations of two excipients. The screening revealed that a wide array of chemicals and coexcipients could modulate intestinal absorption. In particular, the chemical polyethyleneimide increased the transport of Alexa-488-oxytocin, and was selected for pharmacokinetic studies in pigs. Sampling of the pigs' serum after the delivery of the formulation to the pigs' duodenum showed that the absolute oral bioavailability of oxytocin-polyethyleneimide was 
11.3 times higher than oxytocin alone. Also, the formulation did not induce significant loss of epithelial-cell barrier function, neither during in vitro incubation in the robotic culture system nor in in vivo porcine tissue.

Although robotic facilities can be a considerable expenditure, the automation of liquid handling and the analysis of thousands of samples per day can lead to greater efficiency and to time and cost savings. For example, Traverso and colleagues used female porcine explants to assess the expression of native intestinal transporters and of metabolizing enzymes. However, there is a growing body of evidence suggesting that formerly regarded inert excipients have the potential to influence the bioavailability of oral drugs ${ }^{10,11}$, and that drug bioavailability depends on a number of key pharmacokinetic differences between males and females ${ }^{12}$. The robotic explant system could thus be used to assess potential sex differences in the effects of excipients on intestinal absorption, owing to their interaction with drug transporters ${ }^{13}$. Overall, Traverso and co-authors' high-throughput drug-screening system of intestinal drug absorption can facilitate the optimization and formulation of poorly soluble drugs, and help understand the modulation of gene expression, tissue toxicity and enteroendocrine functions.

Abdul W. Basit, Christine M. Madla and Francesca, K. H. Gavins

Department of Pharmaceutics, UCL School of Pharmacy, University College London, 29-39 Brunswick Square, London, WC1N 1AX, United Kingdom.

e-mail: a.basit@ucl.ac.uk; christine.madla.16@ucl.ac.uk; francesca.gavins.13@ucl.ac.uk

\section{References}

1. Vertzoni, M. et al. Eur. J. Pharm. Sci. 134, 153-175 (2019).

2. Osakwe, O. Preclinical In Vitro Studies: Development and Applicability in Social Aspects of Drug Discovery, Development and Commercialization (1st edition, Academic Press, 2016).

3. Sun, H. et al. Exp. Op. Drug Metabol. Toxicol. 4, 395-411 (2008).

4. Von Erlach, T. et al. Nat. Biomed. Eng. , XXX-YYY (2020).

5. Groenen, M. A. M. et al. Nature 491, 393-398 (2012).

6. Hatton, G. B. et al. J. Pharm. Sci. 104, 2747-2776 (2015).

7. Koziolek, M. et al. Eur. J. Pharm. Sci. 134, 31-59 (2019).

8. Hubatsch, I., Ragnarsson, E. G. E. \& Artursson, P. Nat. Protoc. 2, 2111-2119 (2007).

9. Bergström, C. A. S. et al. J. Med. Chem. 46, 558-570 (2003).

10. Flanagan, T. Eur. J. Pharm. Biopharm. 141, 130-138 (2019).

11. Mai, Y. et al. Pharmaceutics. doi: 10.3390/pharmaceutics11050228 (2019).

12. Karp, N. A. \& Reavey, N. Br. J. Pharmacol. 176, 4107-4118 (2019).

13. Stillhart, C. et al. Eur. J. Pharm. Sci. doi: 10.1016/j.ejps.2020.105280 (2020)

14. Drucker, D. J. Nat. Rev. Drug. Discov. doi: 10.1038/s41573-0190-0053-0 (2019).

15. Rehmani, S. \& Dixon, J.E. Peptides. 100, 24-35 (2018).

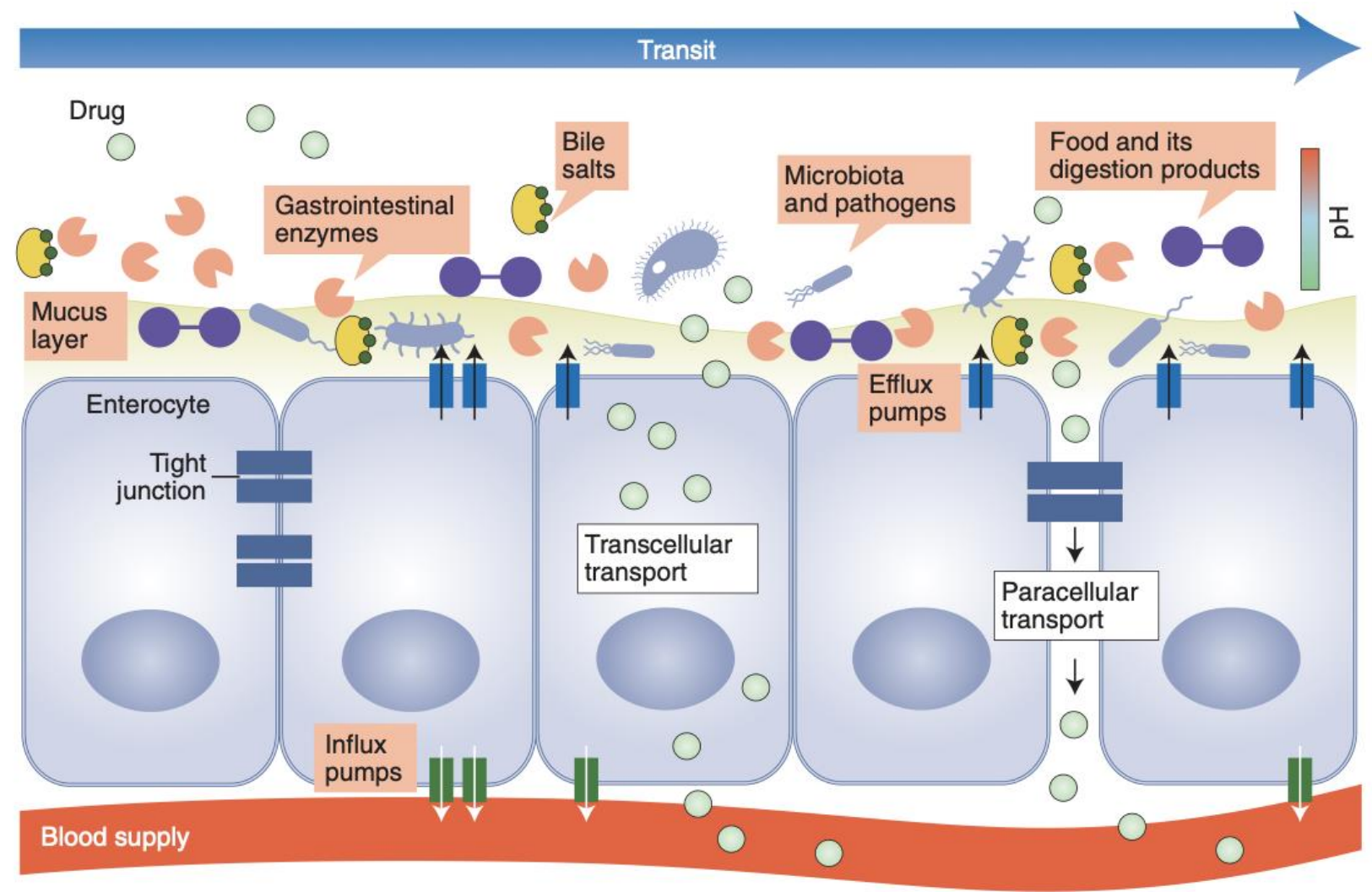

Fig. 1 | The human intestinal barrier. The human gut comprises tight junctions between enterocytes, uptake transporters and efflux transporters, and a mucus layer with metabolizing enzymes, bile salts, and microbiota and pathogens. Figure adapted with permission from refs. ${ }^{14,15}$, Springer Nature Ltd. 

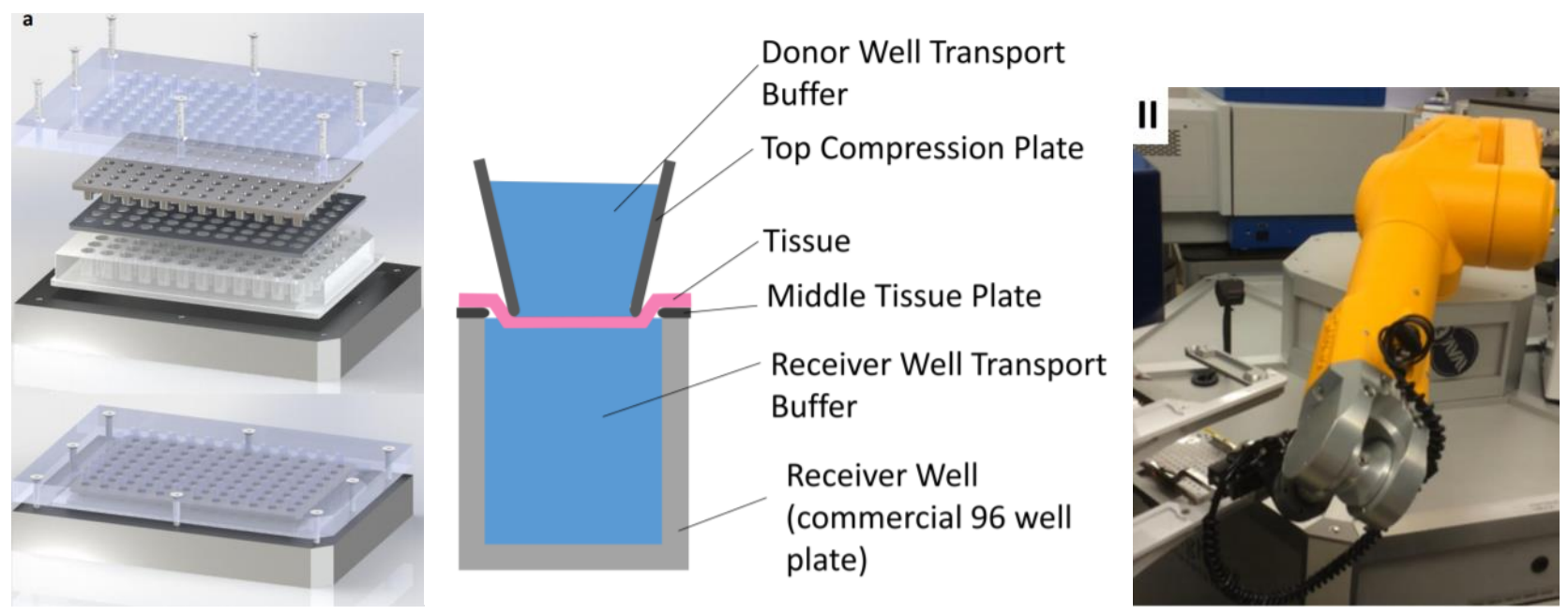

Fig. 21 | A whole-tissue culture system for the screening of oral drugs. a, 96-well device, consisting of three plates, with porcine intestinal explant (not shown) enclosed and sealed off between the upper (light grey) and middle (dark grey) plates. A case (blue) permits adjusting the system's pressure so as to maintain its position. $\mathbf{b}$, A cross-section of a well in the device, showing the donor and receiver containers, separated by the porcine intestinal explant on top of the middle plate. c, Six-axis robotic arm in the process of transferring a plate between different stations. Figure adapted with permission from ref. ${ }^{4}$, Springer Nature Ltd. 